\title{
Constraints on Skyrme Equations of State from Properties of Doubly Magic Nuclei and Ab-Initio Calculations of Low-Density Neutron Matter
}

\author{
B. Alex Brown ${ }^{1}$ and A. Schwenk $k^{2,3}$ \\ ${ }^{1}$ National Superconducting Cyclotron Laboratory and Department of Physics and Astronomy, \\ Michigan State University, East Lansing, Michigan 48824-1321, USA \\ ${ }^{2}$ ExtreMe Matter Institute EMMI, GSI Helmholtzzentrum für Schwerionenforschung GmbH, 64291 Darmstadt, Germany \\ ${ }^{3}$ Institut für Kernphysik, Technische Universität Darmstadt, 64289 Darmstadt, Germany
}

\begin{abstract}
We use properties of doubly-magic nuclei and ab-initio calculations of low-density neutron matter to constrain Skyrme equations of state for neutron-rich conditions. All of these properties are consistent with a Skyrme functional form and a neutron-matter equation of state that depends on three parameters. With a reasonable range for the neutron-matter effective mass, the values of the two other Skyrme parameters are well constrained. This leads to predictions for other quantities. The neutron skins for ${ }^{208} \mathrm{~Pb}$ and ${ }^{48} \mathrm{Ca}$ are predicted to be $0.182(10) \mathrm{fm}$ and $0.173(5) \mathrm{fm}$, respectively. Other results including the dipole polarizability are discussed.
\end{abstract}

PACS numbers: 21.10.Dr, 21.30.Fe, 21.60.Jz, 21.65.-f

The properties of the neutron equation of state (EOS) are important for understanding neutron skins and neutron stars [1], 2], 3]. Recently an extensive study was made of the constraints on Skyrme energy-density functionals (EDFs) provided by the properties of nuclear matter [4]. The standard form and the parameters of the Skyrme functional are given in [4]. Out of several hundred Skyrme EDFs, the 16 given in Table VI of [4] called the CSkP set best reproduced a selected set of empirical nuclear matter properties. Five of these were eliminated [4] since they gave transitions to spin-ordered matter around densities of $\rho=0.25 \mathrm{fm}^{-3}$. One of the remainder (LNS) was unstable for finite nuclei. The remaining 10 are those given in Table I and labeled with their name and order in Table VI of [4]. To this list we add the commonly used SLy4 [5] and SkM* [6] functionals. These 12 EDFs cover a reasonable range of values for the symmetric-nuclear-matter effective mass $\left(\mathrm{m}^{*} / \mathrm{m}\right.$ $=0.70-1.00)$ and incompressibility $\left(K_{m}=212-242 \mathrm{MeV}\right)$ as compared to values extracted from the energy of the giant monopole resonances $\left(K_{m}=217-230 \mathrm{MeV}\right)$ [7].

In 8 these $12 \mathrm{EDFs}$ were refined by a fit to properties of the doubly magic nuclei ${ }^{16} \mathrm{O},{ }^{24} \mathrm{O},{ }^{34} \mathrm{Si}{ }^{40} \mathrm{Ca},{ }^{48} \mathrm{Ca}$, ${ }^{48} \mathrm{Ni},{ }^{68} \mathrm{Ni},{ }^{88} \mathrm{Sr},{ }^{100} \mathrm{Sn},{ }^{132} \mathrm{Sn}$, and ${ }^{208} \mathrm{~Pb}$. The properties included binding energies, single-particle energies [9], root-mean-square (rms) charge radii and rms neutron radii. The most experimentally uncertain of these are the rms neutron radii. With a fixed value for the neutron rms radius of ${ }^{208} \mathrm{~Pb}$, the $t_{0}, t_{1}, t_{2}, t_{3}, x_{0}, x_{3}$ and $W$ (the spin-orbit strength) Skyrme parameters are well determined.

When the neutron skin of ${ }^{208} \mathrm{~Pb}$ is allowed to vary over the range of $R_{n p}=\sqrt{\left\langle r_{n}^{2}\right\rangle}-\sqrt{\left\langle r_{p}^{2}\right\rangle}=0.16$ to 0.24 $\mathrm{fm}$, all of the EDFs cross at a value of the neutron density of about $0.10 \mathrm{fm}^{-3}$ with a value of $E / N=11.3(8) \mathrm{MeV}$ 8]. The slope at this point depends on the neutron skin [1], [8]. The resulting neutron EOS are shown here in
Fig. 1. They are compared with the upper and lower values of the range from next-to-next-to-next-to-leading order $\left(\mathrm{N}^{3} \mathrm{LO}\right)$ calculations of neutron matter [10] (dashed lines). We observe that the Skyrme EOS obtained with a neutron skin of $0.20 \mathrm{fm}$ are in best agreement with the theoretical $\mathrm{N}^{3} \mathrm{LO}$ range.

In this Letter, we follow up on this observation and investigate as additional constraints the results from abinitio calculations of neutron matter. We simultaneously fit the experimental properties of doubly-magic nuclei and the theoretical properties of low-density neutron matter. We do not include any data on the neutron radii of nuclei. Rather as a result of the simultaneous fit we will be able make predictions for the neutron EOS and properties associated with it such as the neutron radii.

We include theoretical data points for the neutronmatter energy at three densities $\rho_{n}=0.01,0.02$, and $0.04 \mathrm{fm}^{-3}$. The range for the energy per particle is based on the recent $\mathrm{N}^{3} \mathrm{LO}$ calculations including two-, threeand four-nucleon interactions in chiral effective field theory (EFT) [10], where the $\mathrm{N}^{2} \mathrm{LO}$ energies and the results with renormalization-group-evolved interactions [11], 3] are also within this range. In addition, we include in the energy range the results of first Quantum Monte Carlo (QMC) calculations with local chiral EFT interactions at NLO and $\mathrm{N}^{2} \mathrm{LO}$ [12] (without three-nucleon forces, which are small at low densities). At the lowest density point, $\rho_{n}=0.01 \mathrm{fm}^{-3}$, the energy range is

$$
[E / N]\left(\rho_{n}=0.010 \mathrm{fm}^{-3}\right)=3.00(13) \mathrm{MeV},
$$

which overlaps with the results from NLO lattice simulations that yield around $3.1 \mathrm{MeV}[13]$. At the second point, $\rho_{n}=0.02 \mathrm{fm}^{-3}$, the $\mathrm{N}^{3} \mathrm{LO}$ range is $E / N=4.14$ $4.34 \mathrm{MeV}$ and the chiral QMC range is $4.39-4.60 \mathrm{MeV}$. These overlap with the variational calculations of $\mathrm{Ak}$ mal et al. based on the Argonne $v_{18} \mathrm{NN}$ and the UIX $3 \mathrm{~N}$ potentials that give $4.35-4.45 \mathrm{MeV}$, where the range is due to including boost corrections [14]. In addition, 


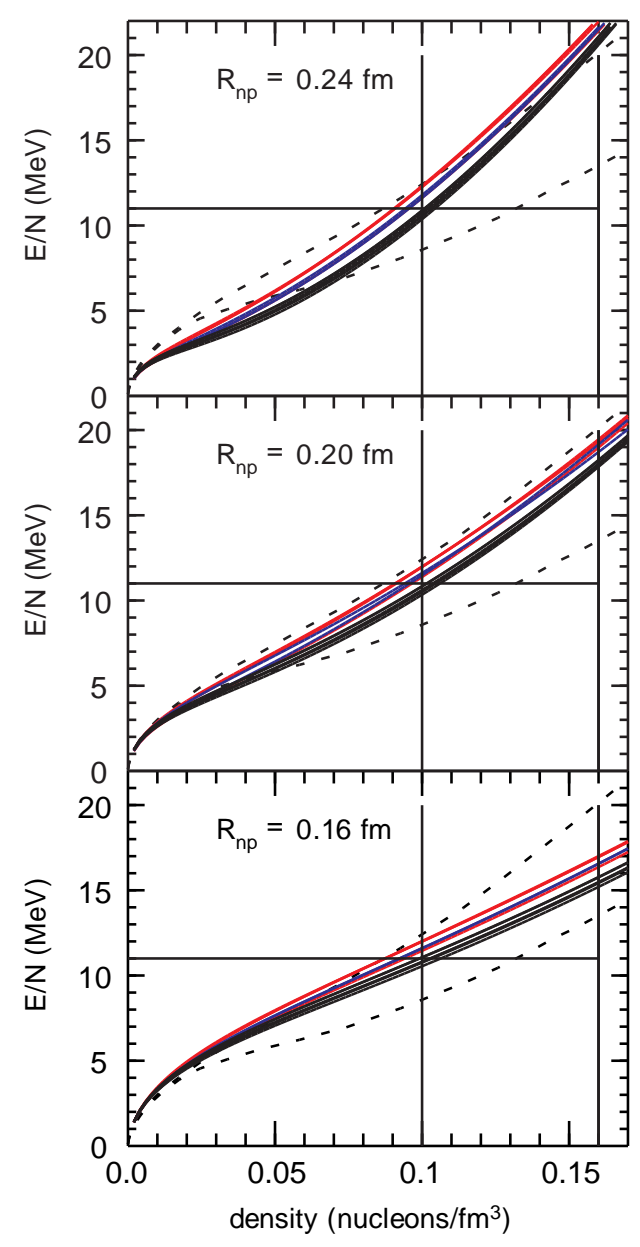

FIG. 1. The neutron EOS with $m_{n}^{*} / m=0.90$ and with $R_{n p}$ fixed at $0.16,0.20$ and $0.24 \mathrm{fm}$ compared to the $\mathrm{N}^{3} \mathrm{LO}$ neutron matter band from [10] (dashed lines). The black lines are those with symmetry nuclear matter values of $m^{*} / m \approx 1.0$ and the red lines with $m^{*} / m=0.70-0.85$. The blue lines are for SLy4 and SkM*.

we consider the Auxiliary Field Diffusion Monte Carlo (AFDMC) results based on the Argonne $v_{8}^{\prime} \mathrm{NN}$ and the same UIX $3 \mathrm{~N}$ forces [15]. Extrapolating these from their lowest density result at $\rho_{n}=0.04 \mathrm{fm}^{-3}$ using their fit gives around 4.6 MeV. Therefore, we adopt as a conservative range for the energy per particle at $\rho_{n}=0.02$ $\mathrm{fm}^{-3}$

$$
[E / N]\left(\rho_{n}=0.020 \mathrm{fm}^{-3}\right)=4.37(23) \mathrm{MeV} .
$$

At the density $\rho_{n}=0.04 \mathrm{fm}^{-3}$, the combined chiral EFT range is $5.40-6.65 \mathrm{MeV}$, which overlaps with the results of Akmal et al. that give 6.23-6.45 MeV, whereas the AFDMC results are 6.79(1) MeV. Therefore, we take

$$
[E / N]\left(\rho_{n}=0.030 \mathrm{fm}^{-3}\right)=6.1(7) \mathrm{MeV} .
$$

Starting with the parameters given in Table VI of [4] we refit $t_{0}, t_{1}, t_{2}, t_{3}, x_{0}, x_{3}$ and $W$ to the doubly-magic

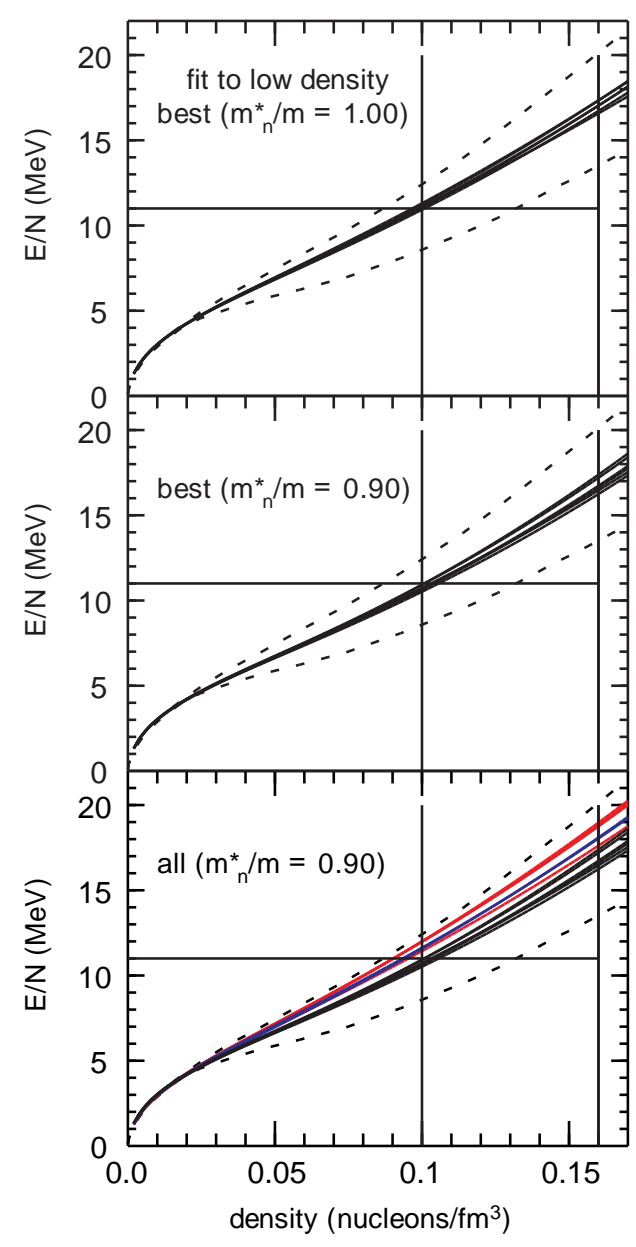

FIG. 2. The neutron EOS from Skyrme fits that include points from ab-initio calculations of low-density neutron matter, compared to the $\mathrm{N}^{3} \mathrm{LO}$ neutron matter band from [10] (dashed lines). The black lines are those with symmetry nuclear matter values of $\mathrm{m}^{*} / m \approx 1.0$ and the red lines with $m^{*} / m=0.70-0.85$. The blue lines are for SLy 4 and SkM*.

data and these three theoretical low-density neutron EOS values. There is no constraint on the neutron skin in the fit. The $t_{0}, t_{1}, t_{2}, t_{3}, x_{0}, x_{3}$ and $W$ Skyrme parameters are all well determined by the fit. The EOS results for the 12 EDFs are shown in the bottom panel of Fig. 2 with the numerical results for the parameters given in Table I. The six "best-fit" results corresponding to those with a symmetric-nuclear-matter effective mass near unity are shown in the middle panel of Fig. 2. Inclusion of the theoretical points for low density does not give a significant increase in the $\chi^{2}$ for the fit compared to those from fits to the nuclear data alone [8]. We find that the theoretical points for low density can be reproduced within their error bars with all 12 of the EDFs and with similar good results for the properties of doubly-magic nuclei. Furthermore, the addition of the low-density points is enough to constrain the Skyrme parameters needed for 
TABLE I. Properties of the fitted Skyrme functionals. The effective mass $m_{n}^{*} / m$ in neutron matter at $\rho_{n}=0.10$ fm ${ }^{-3}$ is constrained to be 0.9 in the first part of the table and 1.0 in the second part. The symmetry energy $J$, its density derivative $L$, the symmetry-energy incompressibility $K_{s}$, the symmetric-nuclear-matter incompressibility $K_{m}$ and effective mass $m^{*} / m$ are evaluated at $\rho=0.16 \mathrm{fm}^{-3}$. The mean value is from the entire set, and the "best" value (b) is for the six cases that give the best fit to the data.

\begin{tabular}{|c|c|c|c|c|c|c|c|c|c|c|c|c|c|c|}
\hline name & & $\sigma$ & $m_{n}^{*} / m$ & $\chi^{2}$ & $\begin{array}{c}K_{m} \\
(\mathrm{MeV})\end{array}$ & $m^{*} / m$ & $\begin{array}{c}a_{n} \\
(\mathrm{MeV} \\
\left.\mathrm{fm}^{3}\right)\end{array}$ & $\begin{array}{c}b_{n} \\
(\mathrm{MeV} \\
\left.\mathrm{fm}^{3 \gamma}\right) \\
\end{array}$ & $\begin{array}{c}d_{n} \\
(\mathrm{MeV} \\
\left.\mathrm{fm}^{5}\right) \\
\end{array}$ & $\begin{array}{c}J \\
(\mathrm{MeV})\end{array}$ & $\begin{array}{c}L \\
(\mathrm{MeV})\end{array}$ & $\begin{array}{c}K_{s} \\
(\mathrm{MeV})\end{array}$ & $\begin{array}{c}R_{n p} \\
(\mathrm{fm}) \\
{ }^{208} \mathrm{~Pb}\end{array}$ & $\begin{array}{c}R_{n p} \\
(\mathrm{fm}) \\
{ }^{48} \mathrm{Ca}\end{array}$ \\
\hline KDE0v1 & s3 & $1 / 6$ & 0.90 & 1.81 & 216 & 0.79 & -455 & 422 & 145 & 34.9 & 61 & -130 & 0.192 & 0.172 \\
\hline NRAPR & s6 & 0.14 & 0.90 & 2.60 & 225 & 0.85 & -534 & 509 & 133 & 35.1 & 61 & -142 & 0.193 & 0.178 \\
\hline Ska25 & s7 & 0.25 & 0.90 & $0.91(\mathrm{~b})$ & 219 & 0.99 & -360 & 328 & 122 & 32.5 & 51 & -138 & 0.176 & 0.170 \\
\hline Ska35 & s8 & 0.35 & 0.90 & $0.80(\mathrm{~b})$ & 244 & 1.00 & -317 & 315 & 123 & 32.8 & 54 & -144 & 0.180 & 0.172 \\
\hline SKRA & s9 & 0.14 & 0.90 & 1.64 & 212 & 0.79 & -504 & 463 & 138 & 33.7 & 55 & -139 & 0.181 & 0.172 \\
\hline SkT1 & s10 & $1 / 3$ & 0.90 & $0.84(\mathrm{~b})$ & 242 & 0.97 & -324 & 326 & 124 & 33.3 & 56 & -140 & 0.183 & 0.172 \\
\hline SkT2 & s11 & $1 / 3$ & 0.90 & $0.86(\mathrm{~b})$ & 242 & 0.97 & -331 & 338 & 125 & 33.5 & 58 & -135 & 0.186 & 0.174 \\
\hline SkT3 & $\mathrm{s} 12$ & $1 / 3$ & 0.90 & $0.80(\mathrm{~b})$ & 241 & 0.98 & -322 & 314 & 134 & 32.7 & 53 & -144 & 0.179 & 0.172 \\
\hline SQMC750 & s15 & $1 / 6$ & 0.90 & 2.41 & 228 & 0.71 & -467 & 447 & 123 & 34.8 & 59 & -148 & 0.190 & 0.176 \\
\hline SV-sym 32 & $\mathrm{~s} 16$ & 0.30 & 0.90 & $0.86(\mathrm{~b})$ & 237 & 0.91 & -335 & 313 & 123 & 32.3 & 51 & -148 & 0.176 & 0.174 \\
\hline SLy4 & s17 & $1 / 6$ & 0.90 & 1.97 & 224 & 0.70 & -450 & 412 & 136 & 34.1 & 56 & -145 & 0.184 & 0.174 \\
\hline $\mathrm{SkM}^{*}$ & $\mathrm{~s} 18$ & $1 / 6$ & 0.90 & 1.69 & 218 & 0.78 & -473 & 450 & 120 & 34.2 & 58 & -139 & 0.187 & 0.175 \\
\hline $\begin{array}{c}\text { mean } \\
\text { mean }(b)\end{array}$ & & & & & & & & & & $\begin{array}{c}33.8(13) \\
32.9(4)\end{array}$ & $\begin{array}{l}56(5) \\
54(4)\end{array}$ & $\begin{array}{l}-138(8) \\
-141(4)\end{array}$ & $\begin{array}{l}0.184(9) \\
0.180(4)\end{array}$ & $\begin{array}{l}0.174(4) \\
0.172(2)\end{array}$ \\
\hline Ska25 & s7 & 0.25 & 1.00 & $0.85(\mathrm{~b})$ & 218 & 0.99 & -386 & 424 & 2 & 32.6 & 48 & -165 & 0.173 & 0.168 \\
\hline Ska35 & s8 & 0.35 & 1.00 & $0.73(\mathrm{~b})$ & 244 & 1.00 & -333 & 419 & -3 & 33.1 & 53 & -165 & 0.179 & 0.171 \\
\hline SkT1 & s10 & $1 / 3$ & 0.99 & $0.76(\mathrm{~b})$ & 241 & 0.97 & -341 & 423 & 0 & 33.4 & 53 & -163 & 0.181 & 0.170 \\
\hline $\mathrm{SkT} 2$ & s11 & $1 / 3$ & 1.00 & $0.76(\mathrm{~b})$ & 230 & 0.97 & -338 & 413 & 4 & 33.1 & 52 & -164 & 0.179 & 0.170 \\
\hline SkT3 & $\mathrm{s} 12$ & $1 / 3$ & 1.00 & $0.73(\mathrm{~b})$ & 241 & 0.98 & -337 & 408 & 3 & 32.8 & 50 & -166 & 0.176 & 0.170 \\
\hline SV-sym 32 & $\mathrm{~s} 16$ & 0.30 & 1.02 & $1.04(\mathrm{~b})$ & 242 & 0.91 & -364 & 450 & -21 & 33.4 & 51 & -176 & 0.178 & 0.173 \\
\hline mean (b) & & & & & & & & & & $33.2(2)$ & $50(3)$ & $-170(7)$ & $0.178(4)$ & $0.171(3)$ \\
\hline overall & & & & & & & & & & $33.1(20)$ & $52(9)$ & $-180(40)$ & $0.182(10)$ & $0.173(5)$ \\
\hline
\end{tabular}

the neutron EOS. This leads to the predictions for EOS properties and neutron skins for ${ }^{208} \mathrm{~Pb}$ and ${ }^{48} \mathrm{Ca}$ given in Table I.

In [4] some of the Skyrme EDFs were eliminated because their neutron-matter effective mass was not less than unity. But as shown in [8] one can include the neutron-matter effective mass in the fit by allowing the $x_{1}$ or $x_{2}$ Skyrme parameters to vary, and this was used to set the neutron-matter effective mass to be 0.9 at a density of $0.10 \mathrm{fm}^{-3}$. In this Letter, we reconsider the value and consequence of the neutron-matter effective mass based on theoretical considerations. At low densities, the neutron-matter effective mass is close to $m_{n}^{*} / m \approx 1.0$. Calculations based on chiral EFT two- and three-nucleon interactions lead to a range $m_{n}^{*} / m=1.0-1.1$ (see Fig. 6 of [1]) and renormalization-group calculations of the Fermi liquid parameters [16] give $m_{n}^{*} / m \approx 1.0$ up to densities $\rho_{n} \lesssim 0.1 \mathrm{fm}^{-3}$. An effective mass close to the bare mass or slightly increased is also expected from the unitary regime of neutron matter at very low densities [17.
To study the importance of the neutron-matter effective mass we refit the six "best-fit" EDFs with a constraint that the neutron matter effective mass is unity. The results shown in the second part of Table I and in the top of Fig. 2. The last line of Table I shows the results and errors that include a variation of 0.9 to 1.1 for the neutron-matter effective mass. The variation in this range leads to a small change in the neutron skins. The largest uncertainty is for the symmetry-energy incompressibility $K_{s}$.

The Skyrme neutron EOS is given by the analytical expression

$$
[E / N](\rho)=a_{n} \rho+b_{n} \rho^{\gamma}+c_{n} \rho^{2 / 3}+d_{n} \rho^{5 / 3}
$$

where $\gamma=1+\sigma$, and $a_{n}, b_{n}, c_{n}$ and $d_{n}$ are constants that depend on the Skyrme parameters. The first term is from the delta-function part that depends on $t_{0}$ and $x_{0}$, the second term is from the density-dependent part that depends on $t_{3}$ and $x_{3}$, the third term is the Fermi-gas kinetic energy, and the fourth term depends on $t_{1}, t_{2}, x_{1}$ 


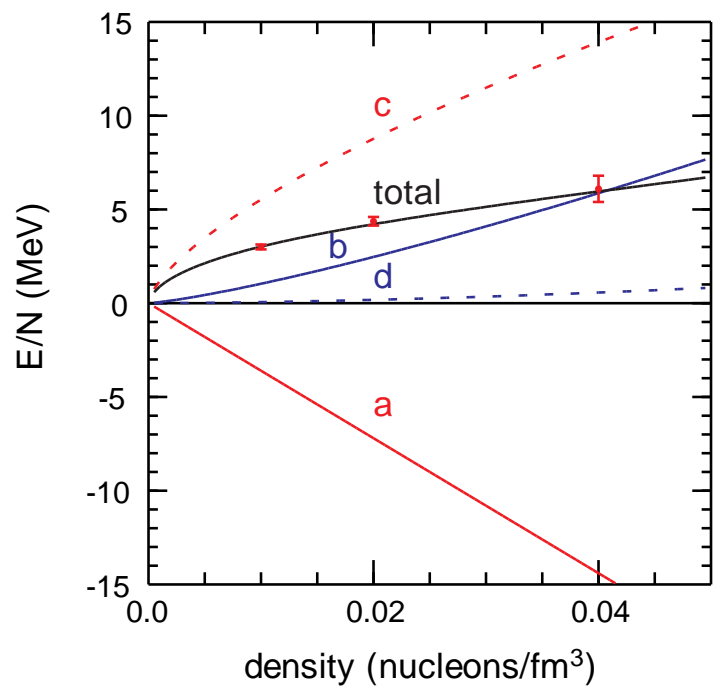

FIG. 3. The neutron EOS at very low density. The total result for the Ska25 fit with a neutron-matter effective mass of 0.9 at a density of $0.10 \mathrm{fm}^{-3}$ is shown (black solid line) together with the separate contributions from the $a_{n}$ (red solid line), $b_{n}$ (blue solid line), $c_{n}$ (red dashed line) and $d_{n}$ (black dashed line) terms of Eq. (4). The theoretical data points are shown by the red points with error bars.

and $x_{2}$. The neutron-matter effective mass is given by

$$
\frac{m_{n}^{*}(\rho)}{m}=\frac{c_{n}}{c_{b}+d_{n} \rho}
$$

The very low-density results for one of the "best-fit" cases with a neutron-matter effective mass of unity are shown in Fig. 3. The neutron EOS is broken down into its four components; $c_{n}=119 \mathrm{MeV} \mathrm{fm}^{2}$ is fixed by the Fermi gas model, $d_{n}$ is relatively small, and the two most important parameters are $a_{n}$ and $b_{n}$. It is remarkable that the theoretical low-density EOS and the properties of doubly-magic nuclei can all be understood with the Skyrme ansatz for the EDF (see also [3] ). The values of $a_{n}$ and $b_{n}$ depend on $\gamma=1+\sigma$, but the resulting neutron matter EOS all give a good fit to the data. There will be an uncertainty in the neutron EOS at high neutron density due to the dependence on $\sigma$ and the effective mass within the Skyrme EDFs. For our set of 12 EDFs and a range of $\left[m_{n}^{*} / m\right]\left(\rho_{n}=0.10 \mathrm{fm}^{-3}\right)=0.9-1.1$, we obtain $[\mathrm{E} / \mathrm{N}]\left(\rho_{n}=0.32\right)=37-51 \mathrm{MeV}$.

It has been shown that the dipole polarizability of ${ }^{208} \mathrm{~Pb}$ is sensitive to the neutron skin of ${ }^{208} \mathrm{~Pb}$ [18] together with other properties of the neutron EOS [19], [20]. The dipole polarizability of ${ }^{208} \mathrm{~Pb}$ was recently measured to be $\alpha_{D}=20.1(6) \mathrm{fm}^{3}$ [21]. The droplet model was used to obtain analytical relationships between $\alpha_{D}$, properties of the symmetry energy and the surface properties of ${ }^{208} \mathrm{~Pb}$ [20]. If we use Eq. (11) from [20] together with the $J$ and $L$ values from the last row of Table I, we obtain $\alpha_{D}=21.3(1.3) \mathrm{fm}^{3}$. If we use Eq. (12) of [20] to obtain $R_{n p}$ from $\alpha_{D}=20.1(6) \mathrm{fm}^{3}$ and $J=33.2(20)$ $\mathrm{MeV}$, we obtain $R_{n p}=0.188 \mathrm{fm}$ with an error of 0.009 coming from Eq. (12) in [20], and an error of 0.011 coming from the error in $J$. Thus, the present predictions are consistent with the measured dipole polarizability from 21].

The ${ }^{208} \mathrm{~Pb}$ neutron skin thickness can also be obtained from the PREX parity-violating electron-scattering experiment of $R_{n p}=0.302 \pm(0.175)_{\exp } \pm(0.026)_{\text {model }} \pm$ $(0.005)_{\text {strange }} \mathrm{fm}$ 22], 23]. A PREX-II experiment has been approved that is expected to reduce the error bar to about $0.06 \mathrm{fm}$. This and the planned parity-violating experiments on ${ }^{48} \mathrm{Ca}$ will be an important test of our predictions.

It will be important to see if any measured property is inconsistent with our predictions within their error range. If so, then a more complex form of the Skyrme functional would be inferred. Also it will be important to carry out our analysis with other density functional forms to see if the conclusions are robust. The present Skyrme EDFs provide a very useful starting point for new supernova EOS.

This work was supported in part by the NSF grant PHY-1068217, the Helmholtz Alliance Program of the Helmholtz Association, contract HA216/EMMI "Extremes of Density and Temperature: Cosmic Matter in the Laboratory", and the ERC Grant No. 307986 STRONGINT. We thank the Institute for Nuclear Theory at the University of Washington for its hospitality.

[1] B. A. Brown, Phys. Rev. Lett. 85, 5296 (2000); S. Typel and B. A. Brown, Phys. Rev. C 64, 027302 (2001).

[2] A. W. Steiner, J. M. Lattimer, and E. F. Brown, Astrophys. J. 765, L5 (2013).

[3] K. Hebeler, J. M. Lattimer, C. J. Pethick, and A. Schwenk, Astrophys. J. 773, 11 (2013).

[4] M. Dutra, O. Louenco, J. S. Sa Martins, A. Delfino, J. R. Stone, and P. D. Stevenson, Phys. Rev. C 85, 035201 (2012).

[5] E. Chabanat, P. Bonche, P. Haensel, J. Meyer, and R. Schaeffer, Nucl. Phys. A 635, 231 (1998).

[6] J. Bartel, P. Quentin, M. Brack, C. Guet, and H. B. Hakansson, Nucl. Phys. A 386, 79 (1982).

[7] L. G. Cao, H. Sagawa and G. Colo, Phys. Rev. C 86, 054313 (2012).

[8] B. A. Brown, arXiv:1308.3664 (2013), and Phys. Rev. Lett. (to be published) (2013).

[9] B. A. Brown, Phys. Rev. C 58, 220 (1998).

[10] I. Tews, T. Krüger, K. Hebeler, and A. Schwenk, Phys. Rev. Lett. 110, 032504 (2013); T. Krüger, I. Tews, K. Hebeler, and A. Schwenk, Phys. Rev. C 88, 025802 (2013).

[11] K. Hebeler and A. Schwenk, Phys. Rev. C 82, 014314 (2010)

[12] A. Gezerlis, I. Tews, E. Epelbaum, S. Gandolfi, K. 
Hebeler, A. Nogga, and A. Schwenk, Phys. Rev. Lett. 111, 032501 (2013).

[13] E. Epelbaum, H. Krebs, D. Lee, and U.-G. Meißner, Eur. Phys. J. A 40, 199 (2009); D. Lee, private communication.

[14] A. Akmal, V. R. Pandharipande, and D. G. Ravenhall, Phys. Rev. C 58, 1804 (1998).

[15] S. Gandolfi, J. Carlson, and S. Reddy, Phys. Rev. C 85, 032801(R) (2012); S. Gandolfi, J. Carlson, S. Reddy, A. W. Steiner, and R. B. Wiringa, arXiv:1307.5815.

[16] A. Schwenk, B. Friman, and G. E. Brown, Nucl. Phys. A 713, 191 (2003).

[17] J. Carlson, S. Gandolfi, and A. Gezerlis, Prog. Theor. Exp. Phys. (2012) 01A209.

[18] P. G. Reinhard and W. Nazarewicz, Phys. Rev. C 81, 051303 (2010).

[19] J. Piekarewicz, B. K. Agrawal, G. Colo, W. Nazarewicz,
N. Paar, P. G. Reinhard, X. Roca-Maza, and D. Vretenar, Phys. Rev. C 85, 041302 (2012).

[20] X. Roca-Maza, M. Centelles, X. Viñas, M. Brenna, G. Colo, B. K. Agrawal, N. Paar, J. Piekarewicz, and D. Vretenar, Phys. Rev. C 88, 024316 (2013).

[21] A. Tamii, I. Poltoratska, P. von Neumann-Cosel, Y. Fujita, T. Adachi, C. A. Bertulani, J. Carter, M. Dozono, H. Fujita, K. Fujita, et al., Phys. Rev. Lett. 107, 062502 (2011).

[22] S. Abrahamyan, Z. Ahmed, H. Albataineh, K. Aniol, D. S. Armstrong, W. Armstrong, T. Averett, B. Babineau, A. Barbieri, V. Bellini, et al. Phys. Rev. Lett. 108, 112502 (2012).

[23] C. J. Horowitz, Z. Ahmed, C.-M. Jen, A. Rakhman, P. A. Souder, M. M. Dalton, N. Liyanage, K. D. Paschke, K. Saenboonruang, R. Silwal, et al., Phys. Rev. C 85, 032501 (2012). 